\title{
From Quasiperiodic Partial Synchronization to Collective Chaos in Populations of Inhibitory Neurons with Delay
}

\author{
Diego Pazó ${ }^{1}$ and Ernest Montbrió ${ }^{2}$ \\ ${ }^{1}$ Instituto de Física de Cantabria (IFCA), CSIC-Universidad de Cantabria, 39005 Santander, Spain \\ ${ }^{2}$ Center for Brain and Cognition, Department of Information and Communication Technologies, \\ Universitat Pompeu Fabra, 08018 Barcelona, Spain
}

(Received 11 November 2015; revised manuscript received 4 May 2016; published 7 June 2016)

\begin{abstract}
Collective chaos is shown to emerge, via a period-doubling cascade, from quasiperiodic partial synchronization in a population of identical inhibitory neurons with delayed global coupling. This system is thoroughly investigated by means of an exact model of the macroscopic dynamics, valid in the thermodynamic limit. The collective chaotic state is reproduced numerically with a finite population, and persists in the presence of weak heterogeneities. Finally, the relationship of the model's dynamics with fast neuronal oscillations is discussed.
\end{abstract}

DOI: 10.1103/PhysRevLett.116.238101

Electrical measurements of brain activity display a broad spectrum of oscillations, reflecting the complex coordination of spike discharges across large neuronal populations [1]. A particularly fruitful theoretical framework for investigating neuronal rhythms is to model networks of neurons as populations of heterogeneous oscillators [2-4]. These models exhibit a prevalent transition from incoherence to partial coherence, when a fraction of the oscillators becomes entrained to a common frequency. As a result, a macroscopic oscillatory mode appears with the same frequency as that of the synchronized cluster $[2,5]$.

Yet, even populations of globally coupled identical oscillators are capable of exhibiting a much wider diversity of complex oscillatory states, see [6] for a recent survey. In general, this is due to the complexity of the coupling functions and of the individual oscillators. A relevant example is the so-called quasiperiodic partial synchronization (QPS), which has been extensively investigated in networks of excitatory leaky integrate-and-fire (LIF) neurons [7-11], as well as in populations of limit-cycle oscillators and phase oscillators [12-18]. In QPS, the network sets into a nontrivial dynamical regime in which oscillators display quasiperiodic dynamics while the collective observables oscillate periodically. Remarkably, the period of these oscillations differs from the mean period of the individual oscillators. As pointed out recently [17], this interesting property of QPS is shared by the collective chaos observed in populations of globally coupled limitcycle oscillators [19-25]. Here, the collective chaotic mode is typically accompanied by microscopic chaotic dynamics at the level of the individual oscillators. However, as noticed in [20], populations of limit-cycle oscillators may also display pure collective chaos without a trace of orbital instability at the microscopic level. In this state, the coordinates of the oscillators fall on a smooth closed curve and no mixing occurs, what points to the existence of collective chaos in populations of oscillators governed by a single phaselike variable.

In this Letter, we uncover the spontaneous emergence of pure collective chaos from QPS via a cascade of perioddoubling bifurcations. Notably, this is found in a simple population of identical integrate-and-fire oscillators with time-delayed pulse coupling, which is thoroughly analyzed within the framework of the so-called Ott-Antonsen theory [26-29]. Moreover, we show that pure collective chaos persists when weak heterogeneities are considered. This suggests that certain forms of irregular collective motion observed in large networks of heterogeneous LIF neurons with delay [30] may already be found for identical neurons.

We investigate a model consisting of a population of $N \gg 1$ neurons, with membrane potentials $\left\{V_{j}\right\}_{j=1, \ldots, N}$. The evolution of $V_{j}$ is governed by the so-called quadratic integrate-and-fire (QIF) model, which obeys the nonlinear differential equation [31-33]

$$
\tau \dot{V}_{j}=V_{j}^{2}+I_{j},
$$

where $\tau$ is the neuron's membrane time constant. When $V_{j}$ reaches the value $V_{p}$, the QIF neuron emits a spike, and $V_{j}$ is reset to $V_{r}$. Thereafter, we consider $V_{p}=-V_{r}=\infty$ [34]. In this case, the model (1) can be exactly transformed to a phase model called theta-neuron $[31,33]$. The external inputs $I_{j}$ have the form

$$
I_{j}=\eta_{j}+J s_{D},
$$

where parameters $\eta_{j}$ determine the dynamics of each uncoupled neuron, $J=0$ : Those neurons with $\eta_{j}<0$ are excitable, whereas neurons with $\eta_{j}>0$ behave as selfsustained oscillators with a period, or interspike interval ISI $_{j}=\pi \tau / \sqrt{\eta_{j}}$. In Eq. (2), the delayed mean activity $s_{D} \equiv s(t-D)$ is defined summing the spikes of all neurons 


$$
s_{D}=\frac{\tau}{N \tau_{s}} \sum_{j=1}^{N} \sum_{k} \int_{t-D-\tau_{s}}^{t-D} \delta\left(t^{\prime}-t_{j}^{k}\right) d t^{\prime} .
$$

In this equation, $t_{j}^{k}$ is the time of the $k$ th spike of the $j$ th neuron, and $\delta(t)$ is the Dirac delta function. We assume the thermodynamic limit $N \rightarrow \infty$, so that a second limit in the temporal window $\tau_{s} \rightarrow 0$ leads to the relationship $s_{D}=\tau r_{D}$, where $r_{D} \equiv r(t-D)$ is the time-delayed firing rate, i.e., the population-averaged number of spikes per unit time. The strength of the interactions is controlled in Eq. (2) by the synaptic weight constant $J$, which can be either positive or negative for excitatory or inhibitory synapses, respectively.

We start performing numerical simulations of an inhibitory $(J<0)$ population of identical neurons with $\eta_{j}=\bar{\eta}>0$. In Figs. 1(a) and 1(c), showing raster plots for two values of $J$, the system exhibits QPS. In fact, the return plots in Figs. 1(b) and 1(d) show a closed line indicating quasiperiodic singleneuron dynamics, see [7]. Remarkably, for certain values of the time delay $D$, see Figs. 1(e) and 1(f), increasing inhibition leads to a different macroscopic state, where neurons exhibit irregular dynamics, whereas the macroscopic dynamics is chaotic, as shown below.

Where and how QPS and collective chaos emerge is investigated next. To this aim, we follow [29] and, using the Ott-Antonsen theory (by means of a Lorentzian ansatz), derive the so-called firing-rate equations (FREs) governing the dynamics of the firing rate $r$, and the population's mean membrane potential $v$. Considering that currents $\eta_{j}$ are distributed according to a Lorentzian distribution of halfwidth $\Delta$, centered at $\bar{\eta}, g(\eta)=(\Delta / \pi)\left[(\eta-\bar{\eta})^{2}+\Delta^{2}\right]^{-1}$, we obtain a system of one ordinary and one delay differential equations [35]
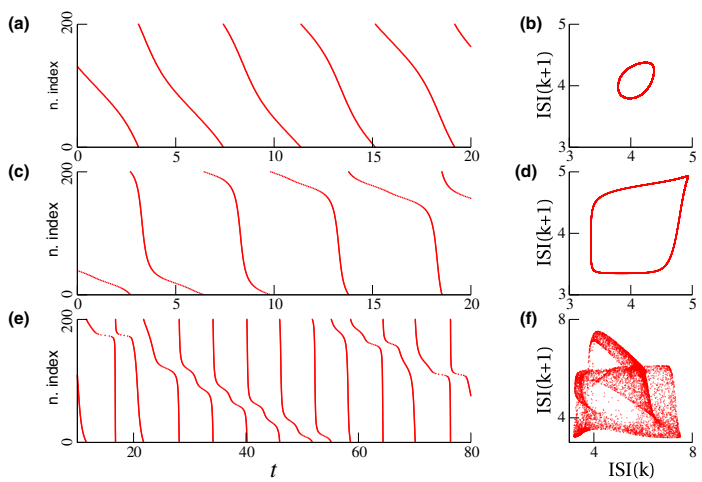

FIG. 1. Quasiperiodic partial synchronization (a)-(d) and collective chaos (e),(f) in an inhibitory network of $N=1000$ identical QIF neurons with $\eta_{j}=\bar{\eta}=\tau=1, \tau_{s}=10^{-3}$, and: (a), (b) $D=2.5, J=-1.65$, (c),(d) $D=2.5, J=-1.85$, and (e),(f) $D=3, J=-3.8$. (a),(c),(e) Raster plots of 200 randomly selected neurons. Dots correspond to firing events, and neurons are indexed according to their firing order. (b),(d),(f) Return plots for $10^{4}$ interspike intervals $\operatorname{ISI}_{j}(k)=t_{j}^{k+1}-t_{j}^{k}$ of an arbitrary neuron $j$.

$$
\begin{gathered}
\tau \dot{r}=\frac{\Delta}{\pi \tau}+2 r v, \\
\tau \dot{v}=v^{2}+\bar{\eta}+J \tau r_{D}-\tau^{2} \pi^{2} r^{2},
\end{gathered}
$$

which exactly describe the macroscopic dynamics of the system in the infinite $N$ limit [36]. Hereafter, we set $\tau=\bar{\eta}=1$ in Eq. (4), without a lack of generality [39].

Figures 2(a), 2(c), and 2(e) display the time series of the population firing rate, using the parameters of Fig. 1 for both the network of spiking neurons (1) and the FREs (4). The attractor of the FREs in Figs. 2(b), 2(d), and 2(f) is in perfect agreement with the global behavior of the population. Figures 1(b) and 1(d) and Figs. 2(a) and 2(c) display the fingerprint of QPS: oscillations of the mean field, with a different period (in the present case, longer) than the individual neurons ISIs. It is noteworthy that the two oscillations shown in Figs. 2(a) and 2(c) have exactly the same period: $T_{1}=2 \mathrm{D}$. This is the consequence of the symmetry of the limit cycle under $v \rightarrow-v$, see Figs. 2(b) and 2(d). Indeed, using Eqs. (4) with $\Delta=0$, one finds that this symmetric cycle is only possible if the period of the oscillation satisfies

$$
T_{m}=\frac{2 D}{m}, \quad \text { with } m=1,3, \ldots
$$

As parameters are varied, the reflection symmetry of the limit cycle breaks down at a period-doubling bifurcation. Moreover, the inset of Fig. 3 shows that this bifurcation is followed by a period-doubling cascade as parameter $J$ is varied, giving rise to a state of collective chaos as that of Fig. 2(f). Remarkably, though the collective dynamics is chaotic, the single-neuron evolution is not. Indeed, as a
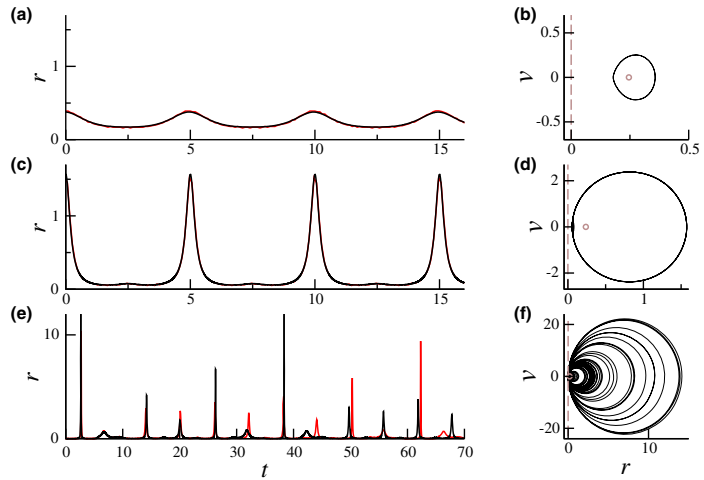

FIG. 2. Macroscopic dynamics of quasiperiodic partial synchronization (a)-(d), and collective chaos (e),(f); same parameters as in Fig. 1 are used. Black curves are obtained integrating the FREs (4), with $\Delta=0$. Red curves in (a),(c),(e) are the firing rates obtained from numerical simulation of the population, Eqs. (1)-(3). Right panels (b),(d),(f): Phase portraits of the FREs. The unstable fixed point (brown circle) and the unstable orbit (brown, dashed line) correspond to incoherence and full synchronization, respectively. The three largest Lyapunov exponents of the chaotic attractor in panel (f) are $\{0.055,0,-0.232\}$. 


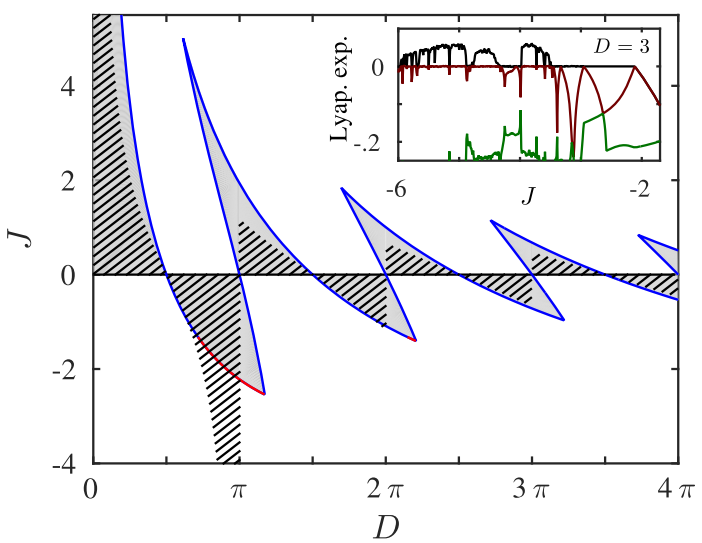

FIG. 3. Stability regions of the incoherent and fully synchronized states for $\Delta=0$. Shaded region: Incoherence is stable. Hatched region: Full synchrony is unstable. Dark gray (blue) and the light gray (red) lines are the loci of subcritical and supercritical Hopf bifurcations of incoherence, respectively. The approximate periodicity of the phase diagram with $D$ stems from the ISI $=\pi$ of the uncoupled neurons. Inset: Three largest (collective) Lyapunov exponents in the range $-6<J<-1.7$ for $D=3$, computed numerically from Eq. (4) using the method in [45]. Note the supercritical Hopf bifurcation at $J_{H}^{(1)}=-2.116 \ldots$ and the accumulation of period-doubling bifurcations at $J \approx 3.5$.

consequence of the mean-field character of the model and its first order kinetics, the firing order of the neurons is preserved (i.e., neuron $j$ always fires just before neuron $j-1$ ), and mixing is not possible.

In the following, we analyze the FREs (4) in detail, which permits us to elucidate why collective chaos is found only in a certain range of delays, and only for inhibitory coupling. For identical neurons, $\Delta=0$, the only fixed point is $\left(r_{s}, v_{s}\right)=\left[\left(J+\sqrt{J^{2}+4 \pi^{2}}\right) /\left(2 \pi^{2}\right), 0\right]$, corresponding to an incoherent state. Its stability can be determined linearizing around the fixed point $r(t)=r_{s}+\delta r e^{\lambda t}$ and $v(t)=\delta v e^{\lambda t}$, and imposing the condition of marginal stability: $\lambda=i \Omega$. We find a family of Hopf instabilities at

$J_{H}^{(n)}=\pi\left(\Omega_{n}^{2}-4\right) \times\left\{\begin{array}{ll}\left(6 \Omega_{n}^{2}+12\right)^{-1 / 2} & \text { for odd } n \\ \left(2 \Omega_{n}^{2}-4\right)^{-1 / 2} & \text { for even } n\end{array}\right.$,

with associated frequencies $\Omega_{n}=n \pi / D$. The line with several cusps depicted in Fig. 3 corresponds to the boundaries of incoherence given by Eq. (6). The blue and red colors indicate the subcritical and supercritical character of the bifurcation, respectively [40]. The stability region of incoherence (shaded) closely resembles that of the Kuramoto model of coupled oscillators, with alternating domains at positive and negative $J$ values as time delay is increased [41-44]. However, the presence of supercritical Hopf bifurcations in some ranges of the inhibitory part of the diagram is a distinct and important feature of the model defined by (1)-(2), as we show below.
We also calculated the stability boundaries of the fully synchronized states, $V_{j}(t)=v(t)$, which are given by the family of functions

$$
J_{c}^{(m)}=2 \cot \left(\frac{D}{m}\right), \quad \text { with } \quad m=1,3,5, \ldots
$$

and by evenly spaced vertical lines at $D=n \pi$, with $n=1,2 \ldots$ [46]. Accordingly, the regions of unstable full synchrony correspond to the hatched regions of the phase diagram in Fig. 3. Note that, for weak coupling, i.e., close to the $J=0$ axis, the phase diagram in Fig. 3 is fully consistent with that of the Kuramoto model with delay [41], as it can be proven applying the averaging approximation to model (1) with $\Delta=0$, see $[5,28]$. Specifically, we observe three qualitatively different regions at small $|J|$ : Incoherence (shaded-hatched), one or more fully synchronized states (white-unhatched), and coexistence between incoherence and full synchrony (shaded-unhatched).

Away from the weak coupling regime, the system displays collective phenomena unseen in the Kuramoto model. Inside the unshaded-hatched region, located below the Hopf curve $J_{H}^{(1)}$, both incoherence and synchronization are simultaneously unstable. Moreover, due to the supercritical character of the Hopf boundary $J_{H}^{(1)}$ in the range $2.250<D<3.684$ [47], QPS emerges as a stable, smallamplitude oscillatory solution - as that of Fig. 2(a) bifurcating from incoherence with period $T=2 D$.

Additionally, QPS can also emerge via the destabilization of full synchronization at $J_{c}^{(1)}$. The simulation of the FREs confirms the prediction of Eq. (7), and allows us to complete a somewhat peculiar picture: The fully synchronous state is a degenerate, infinitely long trajectory along the $v$ axis, and the limit cycle corresponding to QPS emanates from it with an unbounded size-see Fig. 2(d), for a situation not far away from the bifurcation point. In Fig. 4(a), a sketch of the bifurcation diagram (valid for $J<-2.54$ and $D$ around $\pi$ ) is depicted. Stable QPS bifurcates from the fully synchronous state at
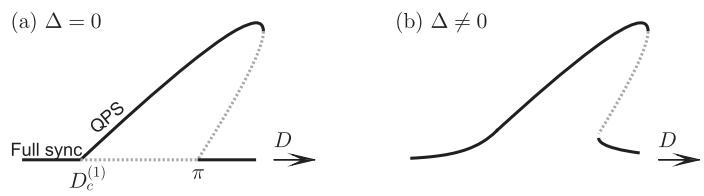

FIG. 4. (a) Sketch of the transition between full synchronization and QPS as $D$ is changed $(J<-2.54, \Delta=0)$; the $y$ axis is an arbitrary coordinate like, e.g., the minimal value attained by the firing rate. Full synchrony undergoes two transcritical bifurcations. The first one, at $D_{c}^{(1)}$, gives rise to QPS which may, eventually, undergo secondary instabilities (not depicted) leading to chaos. At $D=\pi$, full synchrony recovers its stability abruptly. (b) Same sketch for $\Delta \neq 0$. Left transcritical bifurcation evaporates while the right one becomes a saddle-node bifurcation. 


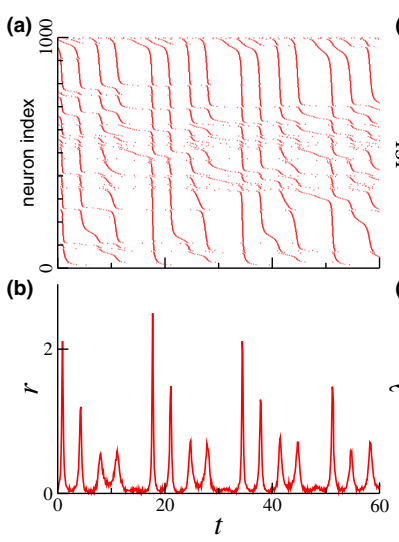

(c)

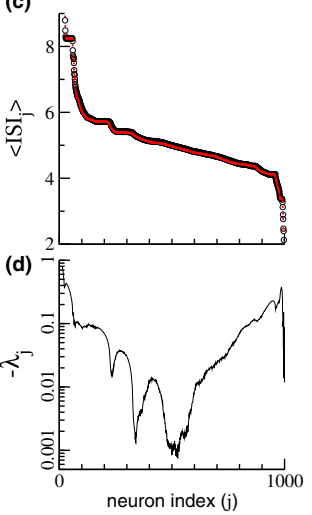

FIG. 5. (a) Raster plot and (b) Time series of the mean firing rate of a population of $N=1000$ heterogeneous $(\Delta=0.025)$ QIF neurons, with inhibitory coupling $(J=-3.8)$ and delay $(D=3.5)$. (c) Time-averaged ISIs of individual neurons vs neuron index (red points) - labels are associated to values of $\eta_{j}$. As a double check, the FREs (4) are simulated for the same parameters and used to drive individual neurons. In this way, we obtain the $\left\langle\mathrm{ISI}_{j}\right\rangle$ depicted by black circles. Note the horizontal segments corresponding to clusters of neurons with identical $\langle$ ISI $\rangle$. (d) Lyapunov exponents $\lambda_{j}$ of individual neurons driven by FREs (4). The $\lambda_{j}$ 's are all negative, while $\lambda_{j}=0$ for the $\Delta=0$ case in Fig. 1(e).

$D_{c}^{(1)}=\arctan (2 / J)<\pi$, through a transcritical bifurcation of limit cycles. Then, in a second transcritical bifurcation at $D=\pi$ (involving unstable QPS), the synchronized state recovers its stability. This scenario implies the existence of a region of bistability between QPS (or collective chaos) and full synchronization for $D>\pi$-in consistence, again, with the supercritical character of the Hopf bifurcation $J_{H}^{(1)}$ for $D<3.684$.

So far, we have concentrated on identical QIF neurons. Our final results concern the robustness of QPS and collective chaos against heterogeneity. In the presence of heterogeneity, full synchronization and QPS cannot be observed, but states reminiscent of them persist, as sketched in Fig. 4(b). Indeed, as the transcritical bifurcation is fragile, the bifurcation originally located at $D=\pi$ is replaced by a saddle-node bifurcation, whereas the other bifurcation at $D=D_{c}^{(1)}$ vanishes.

Regarding collective chaos, Figs. 5(a)-5(c) shows numerical simulations of the heterogeneous QIF neurons (1), with parameter values close to those of Fig. 1(e). We observe, in Fig. 5(c), synchronized clusters at different average ISIs. Using the FREs (4), we checked that (i) the macroscopic infinite- $N$ dynamics of the model is, indeed, chaotic with leading Lyapunov exponents $\{0.013,0,-0.036\}$; (ii) the microscopic dynamics is stable as revealed by the Lyapunov exponents obtained forcing each neuron by Eq. (4), see Fig. 5(d). Interestingly, a similar state was numerically uncovered in [30], and its chaotic nature was attributed to the presence of quenched heterogeneity. However, our conclusion is quite the opposite: the chaotic state in Fig. 5 can be regarded as a perturbed version of the collective chaos in Figs. 1(e) and 2(e), and therefore, heterogeneity is not essential for observing collective chaos.

The fact that the model studied here exhibits nontrivial dynamics precisely for inhibitory coupling - in contrast to the previous studies using LIF neurons [7-11]—deserves to be emphasized. A large body of data demonstrate that brain oscillations in the gamma and fast frequency ranges $(30-200 \mathrm{~Hz})$ are inextricably linked to the behavior of populations of inhibitory neurons [1,48-50]. Moreover, theoretical and computational studies indicate that these oscillations emerge as a consequence of the interplay between inhibition and the significant time delays produced by synaptic processing, see, e.g., [48,51]. Our results add to this body of work, showing that QPS and collective chaos also arise in simple inhibitory populations of phase oscillators with delayed pulse coupling. Plausible values for the synaptic delays are of the order of $D \sim 5 \mathrm{~ms}$, so that the QPS state studied here necessarily has a frequency $f \sim(2 D)^{-1}=100 \mathrm{~Hz}$, corresponding to fast brain oscillations. This is in agreement with the frequency of the oscillations displayed by heuristic firing rate models with fixed time delays and inhibitory coupling [51-54]. Exactly the same range of frequencies is also observed in networks of identical, noise-driven inhibitory neurons with synaptic delays, in the so-called sparsely synchronized state [51,55-57]. Remarkably, sparse synchronization also displays a macroscopic-microscopic dichotomy, similar to that of the QPS and collective-chaos states analyzed here.

The analysis of the thermodynamic limit of the model (1)-(2) by means of the firing-rate equations (4), permits us to dissect macroscopic from microscopic dynamics in that limit. This strategy seems to be particularly useful for investigating collective chaos [19-25] as well as irregular activity states in heterogeneous neuronal ensembles [30,58].

We thank Hugues Chaté for valuable comments. D. P. acknowledges support by MINECO (Spain) under the Ramón y Cajal Programme. We acknowledge support by MINECO (Spain) under Project No. FIS2014-59462-P, and by the European Union's Horizon 2020 research and innovation programme under the Marie Skłodowska-Curie Grant No. 642563.

[1] X.-J. Wang, Physiol. Rev. 90, 1195 (2010).

[2] A. T. Winfree, The Geometry of Biological Time (Springer, New York, 1980).

[3] F. C. Hoppensteadt and E. M. Izhikevich, Weakly Connected Neural Networks. (Spinger Verlag, New York, 1997).

[4] P. Ashwin, S. Coombes, and R. Nicks, J. Math. Neurosci. 6, 2 (2016).

[5] Y. Kuramoto, Chemical Oscillations, Waves, and Turbulence (Springer-Verlag, Berlin, 1984).

[6] A. Pikovsky and M. Rosenblum, Chaos 25, 097616 (2015). 
[7] C. van Vreeswijk, Phys. Rev. E 54, 5522 (1996).

[8] P. K. Mohanty and A. Politi, J. Phys. A 39, L415 (2006).

[9] S. Olmi, A. Politi, and A. Torcini, Europhys. Lett. 92, 60007 (2010).

[10] S. Luccioli, S. Olmi, A. Politi, and A. Torcini, Phys. Rev. Lett. 109, 138103 (2012).

[11] R. Burioni, S. di Santo, M. di Volo, and A. Vezzani, Phys. Rev. E 90, 042918 (2014).

[12] A. Vilfan and T. Duke, Phys. Rev. Lett. 91, 114101 (2003).

[13] M. Rosenblum and A. Pikovsky, Phys. Rev. Lett. 98, 064101 (2007).

[14] A. Pikovsky and M. Rosenblum, Physica (Amsterdam) 238D, 27 (2009).

[15] A. A. Temirbayev, Z. Z. Zhanabaev, S. B. Tarasov, V. I. Ponomarenko, and M. Rosenblum, Phys. Rev. E 85, 015204 (2012).

[16] A. A. Temirbayev, Y. D. Nalibayev, Z. Z. Zhanabaev, V. I. Ponomarenko, and M. Rosenblum, Phys. Rev. E 87, 062917 (2013).

[17] M. Rosenblum and A. Pikovsky, Phys. Rev. E 92, 012919 (2015).

[18] A. Politi and M. Rosenblum, Phys. Rev. E 91, 042916 (2015).

[19] V. Hakim and W. J. Rappel, Phys. Rev. A 46, R7347 (1992).

[20] N. Nakagawa and Y. Kuramoto, Prog. Theor. Phys. 89, 313 (1993).

[21] N. Nakagawa and Y. Kuramoto, Physica (Amsterdam) 75D, 74 (1994).

[22] N. Nakagawa and Y. Kuramoto, Physica (Amsterdam) 80D, 307 (1995).

[23] K. A. Takeuchi, F. Ginelli, and H. Chaté, Phys. Rev. Lett. 103, 154103 (2009).

[24] K. A. Takeuchi, H. Chaté, F. Ginelli, A. Politi, and A. Torcini, Phys. Rev. Lett. 107, 124101 (2011).

[25] W. L. Ku, M. Girvan, and E. Ott, Chaos 25, 123122 (2015).

[26] E. Ott and T. M. Antonsen, Chaos 18, 037113 (2008).

[27] T. B. Luke, E. Barreto, and P. So, Neural Comput. 25, 3207 (2013).

[28] D. Pazó and E. Montbrió, Phys. Rev. X 4, 011009 (2014).

[29] E. Montbrió, D. Pazó, and A. Roxin, Phys. Rev. X 5, 021028 (2015).

[30] S. Luccioli and A. Politi, Phys. Rev. Lett. 105, 158104 (2010).

[31] B. Ermentrout and N. Kopell, SIAM J. Appl. Math. 46, 233 (1986).

[32] P. Latham, B. Richmond, P. Nelson, and S. Nirenberg, J. Neurophysiol. 83, 808 (2000).

[33] E. M. Izhikevich, Dynamical Systems in Neuroscience (MIT Press, Cambridge, MA, 2007).

[34] See Supplemental Material at http://link.aps.org/ supplemental/10.1103/PhysRevLett.116.238101 for details of the numerical implementation.

[35] See Supplemental Material at http://link.aps.org/ supplemental/10.1103/PhysRevLett.116.238101 for the exact derivation of the FREs corresponding to Eqs. (1) and (2).
[36] For identical neurons, the dynamics of the model is degenerate (and described by the Watanabe-Strogatz theory [37]), but the presence of a tiny amount of noise attracts the dynamics to the Lorentzian manifold, making Eq. (4) with $\Delta=0$ asymptotically correct [38]. Accordingly, the initial conditions for the numerical simulations in Figs. 1 and 2 are taken to represent a Lorentzian density with arbitrary values of the center $v$ with width $\pi r: \quad V_{j}(0)=v+\pi r \tan [(\pi / 2)(2 j-N-1) /(N+1)]$, $j=1, \ldots, N$.

[37] S. Watanabe and S. H. Strogatz, Physica (Amsterdam) 74D, 197 (1994).

[38] V. Vlasov, M. Rosenblum, and A. Pikovsky, arXiv: 1601.07170

[39] This can always be achieved (for $\bar{\eta}>0$ ) under rescaling of time $\tilde{t}=t \sqrt{\bar{\eta}} / \tau$, the variables $\tilde{r}=r \tau / \sqrt{\bar{\eta}}, \tilde{v}=v / \sqrt{\bar{\eta}}$, and the parameters $\tilde{J}=J / \sqrt{\bar{\eta}}, \tilde{D}=D \sqrt{\bar{\eta}} / \tau, \tilde{\Delta}=\Delta / \bar{\eta}$.

[40] See Supplemental Material at http://link.aps.org/ supplemental/10.1103/PhysRevLett.116.238101 for the derivation of the amplitude equations close to the Hopf bifurcations using the two-timing method.

[41] M. K. Stephen Yeung and S. H. Strogatz, Phys. Rev. Lett. 82, 648 (1999).

[42] M. Y. Choi, H. J. Kim, D. Kim, and H. Hong, Phys. Rev. E 61, 371 (2000).

[43] E. Montbrió, D. Pazó, and J. Schmidt, Phys. Rev. E 74, 056201 (2006).

[44] W. S. Lee, E. Ott, and T. M. Antonsen, Phys. Rev. Lett. 103, 044101 (2009).

[45] J. D. Farmer, Physica (Amsterdam) 4D, 366 (1982).

[46] See Supplemental Material at http://link.aps.org/ supplemental/10.1103/PhysRevLett.116.238101 for the derivation of the stability boundaries of the fully synchronized state.

[47] Supercriticality is also present for the line $J_{H}^{(3)}$ in a tiny interval above $D=2 \pi$.

[48] X.-J. Wang and G. Buzsáki, J. Neurosci. 16, 6402 (1996).

[49] M. Whittington, R. Traub, N. Kopell, B. Ermentrout, and E. Buhl, Int. J. Psychophysiol. 38, 315 (2000).

[50] M. Bartos, I. Vida, and P. Jonas, Nat. Rev. Neurosci. 8, 45 (2007).

[51] N. Brunel and V. Hakim, Chaos 18, 015113 (2008).

[52] A. Roxin, N. Brunel, and D. Hansel, Phys. Rev. Lett. 94, 238103 (2005).

[53] D. Battaglia, N. Brunel, and D. Hansel, Phys. Rev. Lett. 99, 238106 (2007).

[54] A. Roxin and E. Montbrió, Physica (Amsterdam) 240D, 323 (2011).

[55] N. Brunel and V. Hakim, Neural Comput. 11, 1621 (1999)

[56] N. Brunel and X.-J. Wang, J. Neurophysiol. 90, 415 (2003).

[57] N. Brunel and D. Hansel, Neural Comput. 18, 1066 (2006).

[58] E. Ullner and A. Politi, Phys. Rev. X 6, 011015 (2016). 